\title{
Digital Twin Concept for Aircraft Sensor Failure
}

\author{
Omar HAZBON ALVAREZ ${ }^{\mathrm{a}, 1}$, Luis GUTIERREZ ZEA ${ }^{\mathrm{a}}$, Cees BIL ${ }^{\mathrm{b}}$, \\ Marcello NAPOLITANO ${ }^{c}$ and Mario L. FRAVOLINI ${ }^{\mathrm{d}}$ \\ ${ }^{a}$ Universidad Pontificia Bolivariana, School of Aeronautical Engineering, Medellin, \\ Colombia \\ ${ }^{\mathrm{b}}$ RMIT University, School of Engineering, Melbourne, Australia \\ ${ }^{c}$ West Virginia University, School of Mechanical and Aerospace Engineering, \\ Morgantown, USA \\ ${ }^{d}$ University of Perugia, Department of Engineering, Perugia, Italy
}

\begin{abstract}
The aircraft air data system is used for estimating vital flight parameters for correct and safe aircraft operations. One of the main components in this system is the Pitot tube sensor which is responsible for measuring the total pressure and in conjunction with the static pressure port estimates parameters like altitude, air speed, vertical speed and Mach number. The Pitot tube is prone to failure because of external adverse atmospheric conditions. The current research aims to develop a solution to the aircraft loss of control caused by unreliable and/or faulty airspeed measurements by developing an air data system tolerant to the Pitot sensor failure based on extra sensor measurements and an estimation algorithm that uses a digital twin aircraft dynamic model as a virtual sensor.
\end{abstract}

Keywords. Digital Twin, Flight Safety, Human Factors, Sensor Failure

\section{Introduction}

The Air Data System (ADS) plays a vital role in aircraft operation. The information provided by this device is used by the pilot and other aircraft subsystems for maneuvering and navigating within safe performance boundaries. There is a history of aircraft accidents caused by the failure of one of the ADS sensor, the Pitot tube, for example Air France flight 447 in June 2009, Saratov Airlines flight 6W703 and Lion Air flight 610 in 2018. Table 1 shows a list of some selected non-military aircraft accidents caused by suspected Pitot tube failure since 1973.

The Pitot tube sensor is responsible for airspeed estimation. Under certain atmospheric conditions the sensor becomes covered or blocked with ice, dirt or even ground protection devices and, as a result, the computed airspeed becomes erratic or unreliable. This affects air safety as pilots may not be able to identify the failure and become confused due to unreliable and conflicting warnings. In unmanned air vehicles the situation is also critical because the autopilot is receiving erratic information from the ADS system causing a total loss of control.

\footnotetext{
${ }^{1}$ Corresponding Author, Email: omar.hazbon@upb.edu.co.
} 
Aircraft manufacturers have implemented sensor redundancy with a voting scheme to detect and isolate faulty air data sensor signals. However under certain atmospheric circumstances all sensors might fail at the same time, which is called common mode failures. Common mode failures by caused by cold and humid atmospheric air were icing can block the dynamic and static orifices and drain holes. Water can also accumulate in the probe or pressure lines when the drain hole is obstructed by ice or a foreign object.

Table 1. Civil aircraft accidents caused by Air Data System failure.

\begin{tabular}{|c|c|c|c|}
\hline Date & $\begin{array}{l}\text { Aircraft } \\
\text { Damage }\end{array}$ & Cause & $\begin{array}{l}\text { Casualties } \\
\text { / Injured }\end{array}$ \\
\hline 30 January 1973 & Aircraft total Loss & Ice in Pitot tubes & 0 \\
\hline $\begin{array}{c}1 \text { December } \\
1974\end{array}$ & Aircraft total Loss & Ice in Pitot tubes & 3 \\
\hline 28 July 1984 & Aircraft total Loss & Pitot tube covers not removed & 0 \\
\hline 21 May 1986 & Aircraft total Loss & Ice on Pitot tubes & 0 \\
\hline 2 March 1994 & $\begin{array}{l}\text { Structural } \\
\text { damage }\end{array}$ & Ice on Pitot tubes & 0 \\
\hline 6 February 1996 & Aircraft total loss & Dust or insect debris blocking Pitot tubes orifices & 189 \\
\hline 2 October 1996 & Aircraft total loss & Adhesive tape blocking ADS static port orifice & 70 \\
\hline 10 October 1997 & Aircraft total loss & Ice on Pitot tubes & 74 \\
\hline 7 April 1999 & Aircraft total loss & Ice on Pitot tubes & 6 \\
\hline 17 October 1999 & Aircraft total loss & Ice blocking Pitot tube drain orifices & 0 \\
\hline 3 June 2006 & Aircraft total loss & Obstruction of Pitot tube orifices & 0 \\
\hline $\begin{array}{l}25 \text { February } \\
2009\end{array}$ & Aircraft total loss & Radio Altimeter failure & $9 / 50$ \\
\hline 1 June 2009 & Aircraft total loss & Obstruction of Pitot tube orifices & 228 \\
\hline $\begin{array}{c}11 \text { February } \\
2018\end{array}$ & Aircraft total loss & Ice on Pitot Tubes & 71 \\
\hline 29 October 2018 & Aircraft total loss & ADS Failure & 189 \\
\hline
\end{tabular}

Aircraft automatic control systems are intended to improve flight safety and reduce flight crew workload, however they do rely on the sensor systems working properly. As evident by recent accidents, due to system complexity, sensor failure can lead to sudden and unexpected adverse aircraft behaviour which can leave the flight crew confused and unable to react effectively.

\section{Modelling Virtual Sensors}

The airspeed sensor failure problem has been worked out by means of a Sensor failure detection identification and accommodation (SFDIA) task. SFDIA has been traditionally divided in two steps. The first step involves the detection of the sensor failure and identification (SFDI) of the sensor that is generating the unhealthy signal so the corrupted hardware can be isolated. Sensor failure accommodation (SFA), the second task, consist in the numerical estimation of the airspeed by means of a virtual sensor and the statistical comparison of this value with the sensor (or sensors) signal so the healthy parameter can be selected and used in the flight control system.

\subsection{Model based virtual sensors}

The model-based airspeed estimation approach takes advantage of the well-known aircraft nonlinear dynamic model and measurements from the onboard sensors $[1,2]$. The model state variables are the true air speed, angles of attack and side slip, angular rates, 
Euler attitude angles and the GPS-based aircraft positions. The state control variables rely on thrust force and elevator, rudder, and aileron deflections. The airspeed is implicit in all the 12 equations of the aircraft nonlinear model, however an effort was made to reduce the computational effort by using only the angle of attack equation that is strongly correlated with air speed [3]. This equation is expressed in a form that is suitable for parameter identification with a linear combination of unknown coefficients and known nonlinear function of measured signals. The equation is evaluated at several instants on a time window to set up a linear systems of equations that is solved for the unknown equation coefficients using least squares and taking advantage of previously recorded flight test data. The online estimates of the airspeed at a particular time are based on the online solution of the quadratic equation were the unknown coefficients are substituted by the LS estimate and only the positive value of the airspeed is selected. The results showed that the accuracy of the method is about $5 \%$ of the mean velocity.

\subsection{Model independent virtual sensors}

The model-independent virtual sensors are of great interest because the estimation method can be applied conveniently to any aircraft without information of the vehicle dynamics which most of the time is difficult to obtain. Kalman Filter based estimators and data driven methods based on NN are commonly employed. Napolitano et al. developed model independent virtual sensors by using Extended and Unscented Kalman Filter estimators [3-6]. The data-driven approach of model independent virtual sensors take advantage of artificial neural networks - ANN (Multi-Layer Perceptron - MLP and Extended Minimal Resource Allocating Network - EMRAN) [6].

The MLP ANN was selected for the estimation of airspeed due to its flexibility [6]. The MLP is trained to learn the functional relationships between the airspeed and a set of correlated measurements provided by the other aircraft sensors. The MLP architecture employed consists of one hidden layer with three neurons and a sigmoid activation function. ANN training uses angular rates, linear accelerations, angles of attack, sideslip and roll and angle of pitch as inputs.

The EMRAN ANN architecture allows only the parameters of the most active neurons to be updated, while the others are left unchanged. Essentially, the EMRAN algorithm allocates neurons in order to decrease the estimation error in regions of the state space where the mapping accuracy is poor. This strategy results in a significant reduction of the number of parameters to be updated online, thus reducing the computational burden, and therefore making this architecture particularly suitable for online applications. The procedure followed to train and validate the EMRAN ANN is similar to the MLP ANN with the difference that in initializing the interconnection weights and biases step the network starts without hidden layer neurons based on the three error criteria [6].

\section{Proposed Methodology}

The objective of the project is to develop a new approach to perform SFDIA in case of ADS sensor failure. The SFDIA algorithm estimates the aircraft airspeed and other aircraft parameters using information from gyroscopes and accelerometers. A

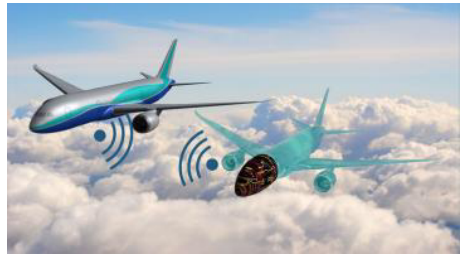

Figure 1. Aircraft digital twin concept. 
Global Positioning System - GPS, a magnetometer and a barometric altimeter are used as aiding sensors for estimation purposes. Sensor data fusion and estimation are done by a Kalman Filter based algorithm assisted by an accurate aircraft dynamic model acting as a virtual sensor referred to as the Aircraft Digital Twin (Figure 1). FDI is carried out automatically by statistical evaluation of the sensor residual signal and accommodation is done automatically by modulating the faulty sensors covariance by giving it less weight during the estimation process. The proposed system is shown in Figure 2.

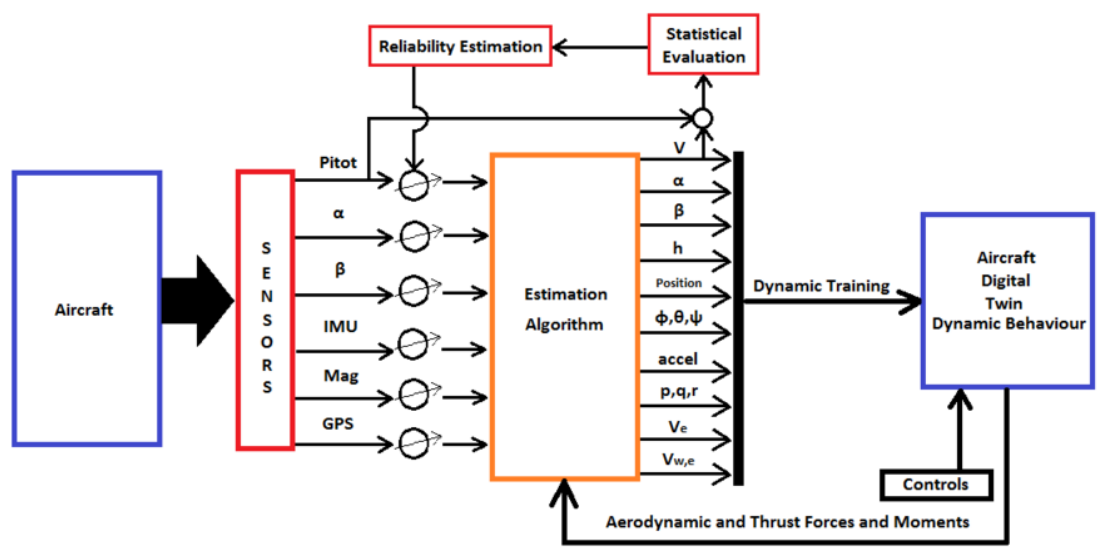

Figure 2. Proposed airspeed estimation scheme.

\subsection{Air Data System}

The ADS models use dynamic pressure, angle of attack, angle of sideslip, atmospheric pressure and temperature as input signals and adds noise and bias in each sensor model to return the measured values. Figure 3 shows the Simulink block diagram of the ADS.

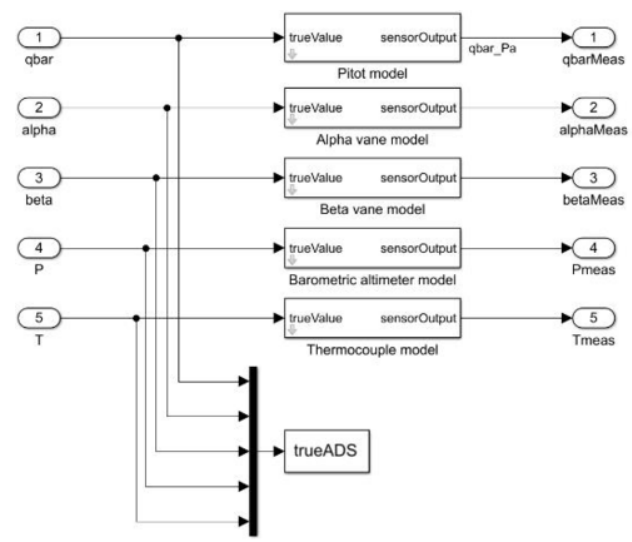

Figure 3. Simulink model of Air Data System.

The Pitot was modelled using as input signal the dynamic pressure trough a zeroorder hold to convert the continuous signal to discrete time. The sensor dynamics was modelled using the discrete state space model to which a white noise and a bias modelled as a random walk was added to obtain the dynamic pressure measured signal. Figure 4 
shows the Simulink block diagram for this sensor. The angle of attack vane sensor was modelled using as input signal the angle of attack to which a white noise and a bias modelled as a random walk is added. The sensor dynamics is modelled as a first order state space model. The signal enters a zero-order hold so a conversion from continuous system to discrete time is carried out before giving as output the measured angle of attack.

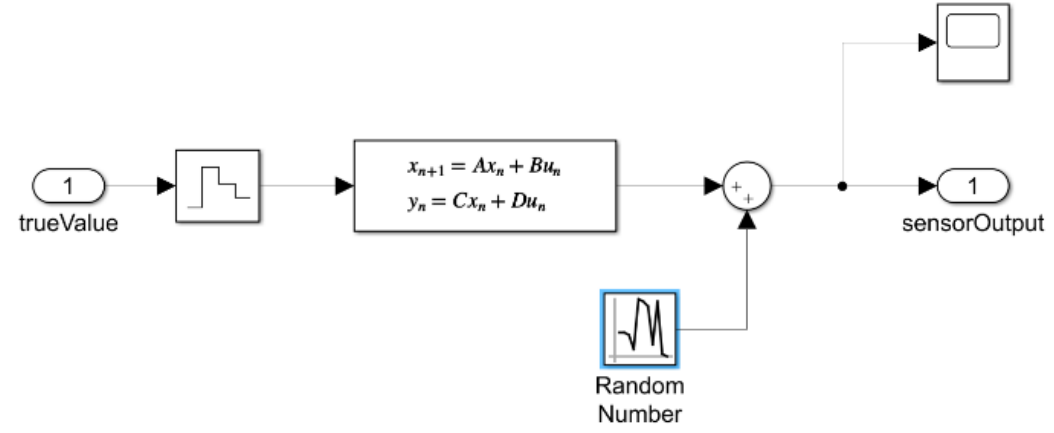

Figure 4. Simulink model of the Pitot sensor.

A similar model is applied to the sideslip angle vane, the barometric altimeter and the temperature probe sensors but using the sideslip angle, the ambient static pressure and the ambient air temperature respectively as inputs signals. Figure 5 shows the Simulink block diagram for these sensors.
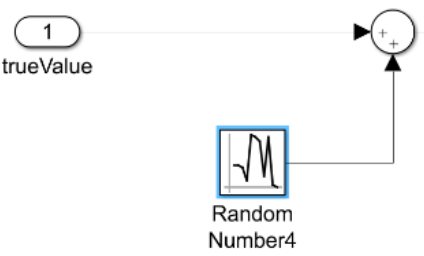

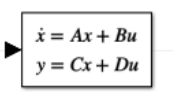

First order model
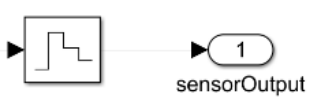

Figure 5. Simulink model of the angle of attack, sideslip, barometric altimeter and temperature sensors.

\subsection{Inertial Measurement Unit (IMU)}

The IMU is composed of 3-axis accelerometers and gyroscopes with angular velocity and linear acceleration as inputs respectively. To each signal a white noise and a bias is added, modelled as a random walk. The noisy and biased signal of each sensor is subjected to a first-order model dynamics in state space form and a zero-order hold to convert the continuous time signal to discrete time before it outputs the measured acceleration and angular velocity. True accelerometer and gyroscope bias signals are taken directly from the random walk. Figures 6 and 7 show the IMU Simulink $₫$ block diagram and each sensor model respectively.

\subsection{Magnetometer}

The magnetometer was modelled using latitude, longitude, altitude and $C_{b}^{e}$ as inputs and returning the Earth's magnetic field vector in the aircraft position and its true bias $b_{m}$. The Simulink model was constructed using a Matlab embedded function block that runs the wrldmagm function. White noise and a bias were modelled as random walks and 
added to the wrldmagm output signal. The noisy and biased signal was passed through a first-order state space model to mimic its dynamics and a zero-order hold to convert from continuous to discrete time. Figure 8 shows the magnetometer Simulink ${ }^{\odot}$ block diagram.
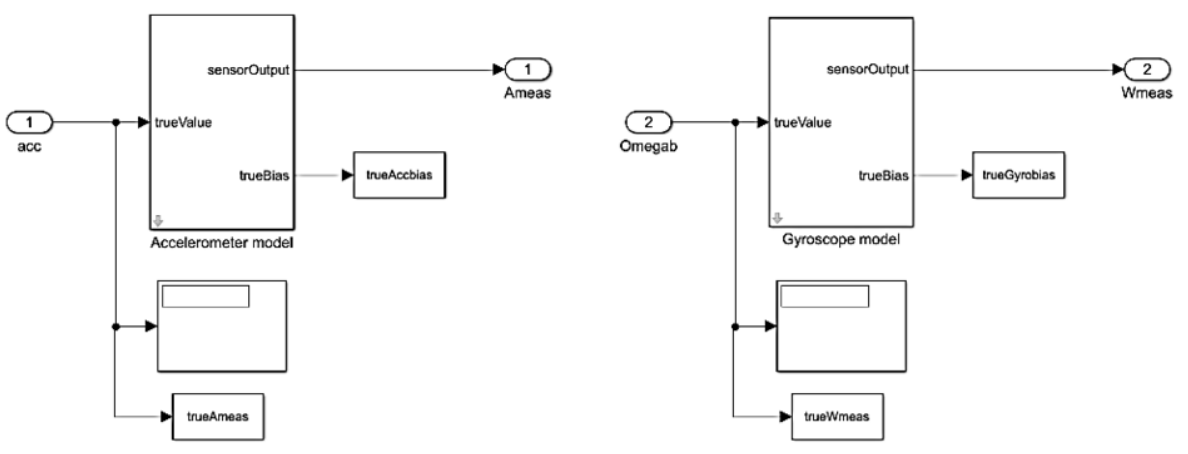

Figure 6. Inertial Measurement Unit Simulink model.

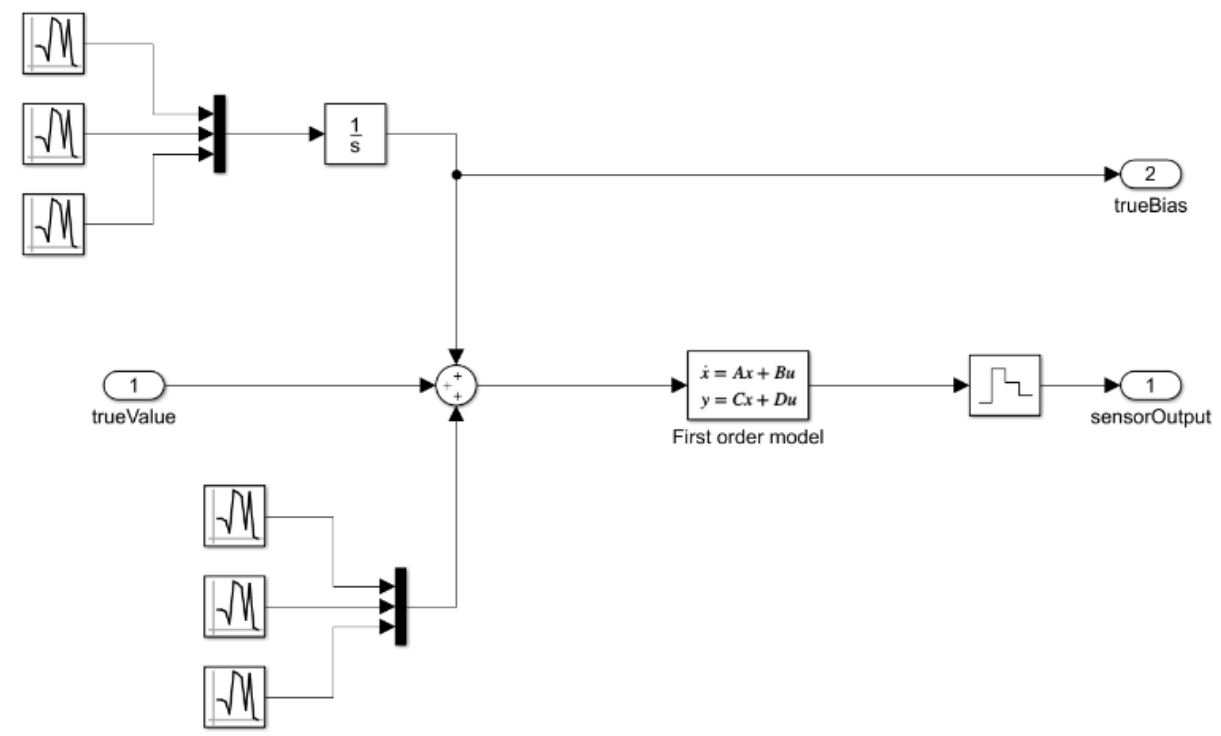

Figure 7. Accelerometers and Gyroscopes Simulink model.

\subsection{Global Positioning System (GPS)}

The GPS model takes the simulation values of latitude, longitude, altitude and velocity as inputs. A bias modelled as a random walk that passes through an integrator is added to each position component. A zero-order hold and a unit delay is applied to the biased signal before the output of the GPS measured position. Figure 9 show the GPS position Simulink $($ block diagram and its sensor model.

The GPS velocity is modelled by adding a bias to each component of the simulation velocity. Then the biased velocity signal is passed through a zero-order hold and a unit delay before giving the GPS measured velocity as output. Figure 10 shows the GPS velocity Simulink $($ block diagram and its sensor model. 


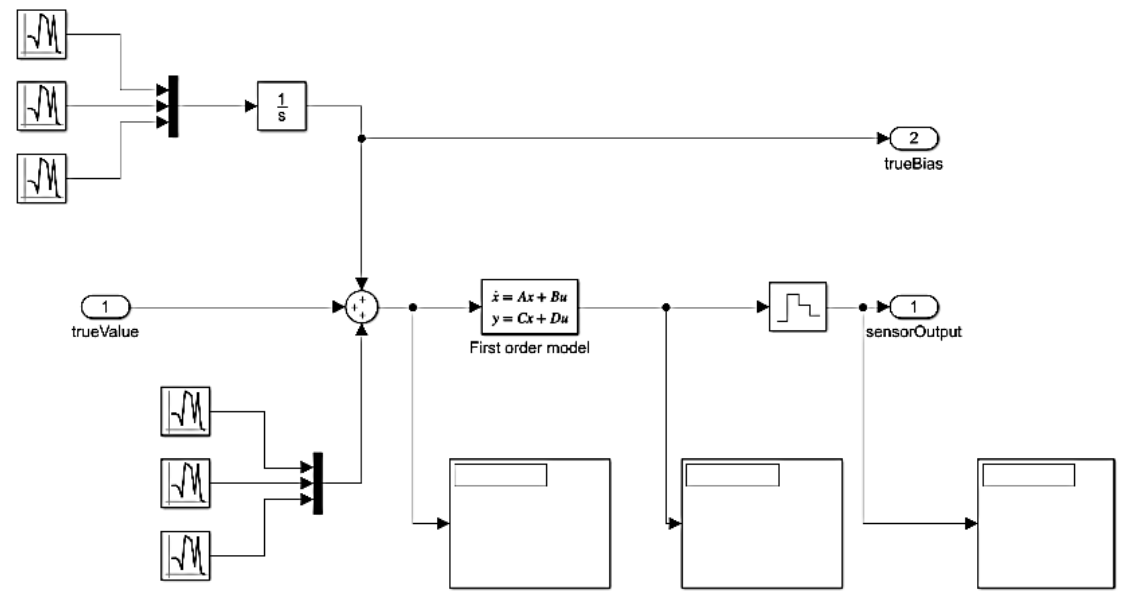

Figure 8. Magnetometer Simulink model.

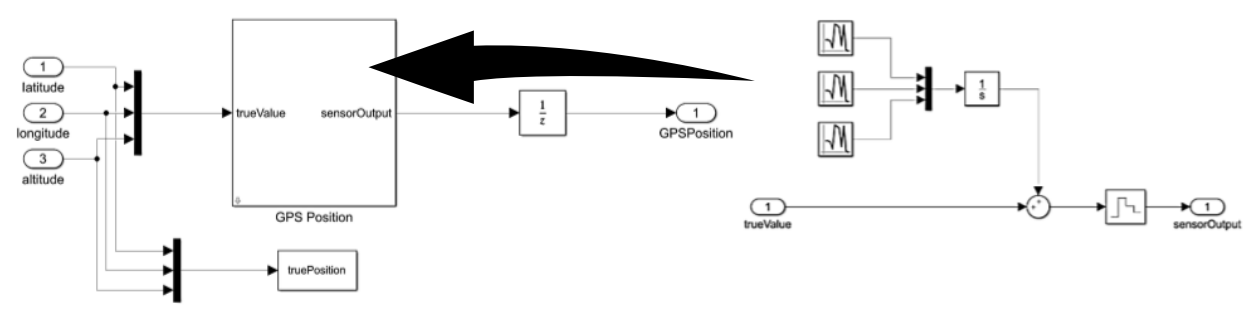

Figure 9. GPS Simulink model (position).

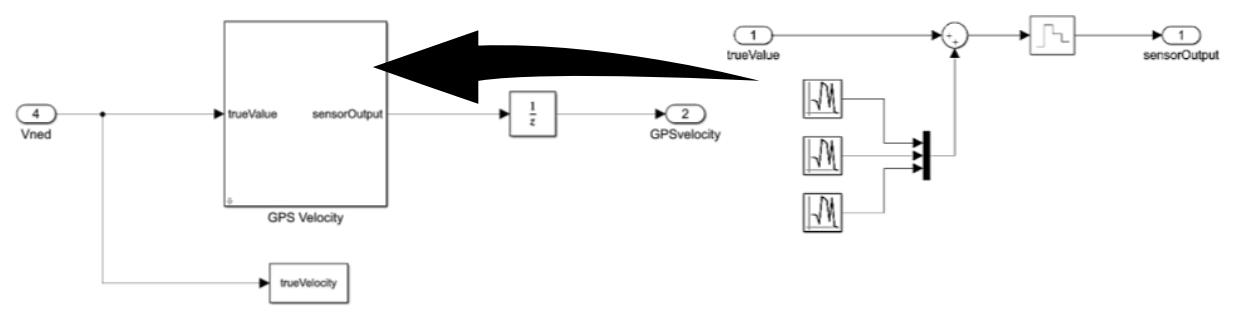

Figure 10. GPS Simulink model (velocity).

\section{Navigation System Extended Kalman Filter}

The navigation system fuses sensor information from the IMU (gyroscopes and accelerometers), global positioning system GPS, a 3 -axis magnetometer and a barometric altimeter in a framework of Extended Kalman Filter with sequential measurement update (SMU-EKF). In this estimation method, the sensor measurements can easily be fused regardless of the number of sensors, sensor update rates, and sensor data dimensions [7].

The core sensor in the estimation algorithm is the IMU, however to increase the accuracy of the estimation a GPS, a 3-axis magnetometer and a barometric altimeter are used as supporting sensors. The filter's estimated state includes the aircraft position, velocity, attitude, accelerometer bias and gyroscope bias. The IMU provides the measured linear acceleration $a_{m}^{b}$ and angular velocity $\Omega_{m}^{b}$. 


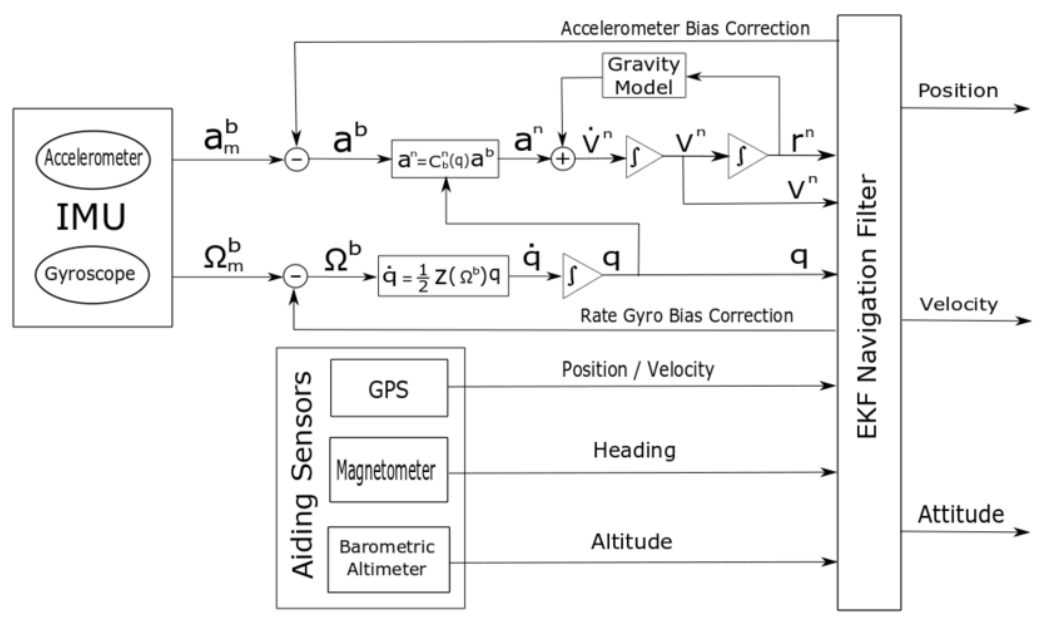

Figure 11. Navigation System Extended Kalman Filter.

The navigation EKF algorithm estimates the IMU sensor bias to correct each measured signal and obtain the unbiased linear acceleration $a^{b}$ and angular velocity $\Omega^{b}$. The acceleration is transformed from the body axis to the navigation frame using the direction cosine matrix $C_{b}^{n}$ to obtain $a^{n}$. Since $a^{n}$ is a specific force, gravity is added to obtain the acceleration in the navigation frame $\dot{V}^{n}$. By integrating twice, the speed $V^{n}$ and position $r^{n}$ in the navigation frame are obtained. As shown in Figure 11, $r^{n}$ is used in conjunction with the world gravitation model to compute the gravity vector. The aircraft attitude $q$ is obtained by integration of the attitude rate $\dot{q}$ which is computed when the angular velocity $\Omega^{b}$ feeds the quaternion kinematical equations.

Figure 12 shows the aircraft velocity estimation results. The aircraft flys north and after $20 \mathrm{~s}$ it turns east 45 degrees and continue level flight. After $120 \mathrm{~s}$ it starts climbing until $180 \mathrm{~s}$ when it levels off. The estimated velocity follows the true velocity accurately during all maneuvers. The estimated downward component of the velocity deviates $1 \mathrm{~m} / \mathrm{s}$ during climb, in other maneuvers the airspeed components deviate $0.15 \mathrm{~m} / \mathrm{s}$.

Figure 13 shows the aircraft attitude estimation. The estimation accurately follows the aircraft attitude true value in all maneuvers, particularly in roll and yaw. At the beginning of the climb there is a deviation of 1 degree and when the aircraft is leveling for cruise there is a deviation of 2 degrees, however in both cases the estimation in pitch converges to the true value in $20 \mathrm{~s}$. This simulation was repeated three times by varying the sensors bias and noise parameters without noticing an effect on the estimation accuracy.

The estimation results described in the previous section were computed without the implementation of the aircraft digital twin. Current research efforts are focused on the integration of the aircraft digital twin with the estimation algorithm described above. Figure 14 shows the integrated navigation system with the aircraft digital twin.

The estimation algorithm described in previous section provide accurate information of $a^{b}$ and $\Omega^{b}$. This values will pass through a first-order filter to simulate its dynamics. In the case of $\Omega^{b}$ the value of its derivative $\dot{\Omega}^{b}$ can be extracted. These values can be used together with the propulsion force $F_{t}^{b}$ and moment $M_{t}^{b}$, the aircraft's controls, inertia matrix $I_{b}$, mass $m$ and CG location to feed the equations of motion (1) and (2) to obtain the measured aircraft's aerodynamic force $F_{a}^{b}$ and moment $M_{a}^{b}$. 

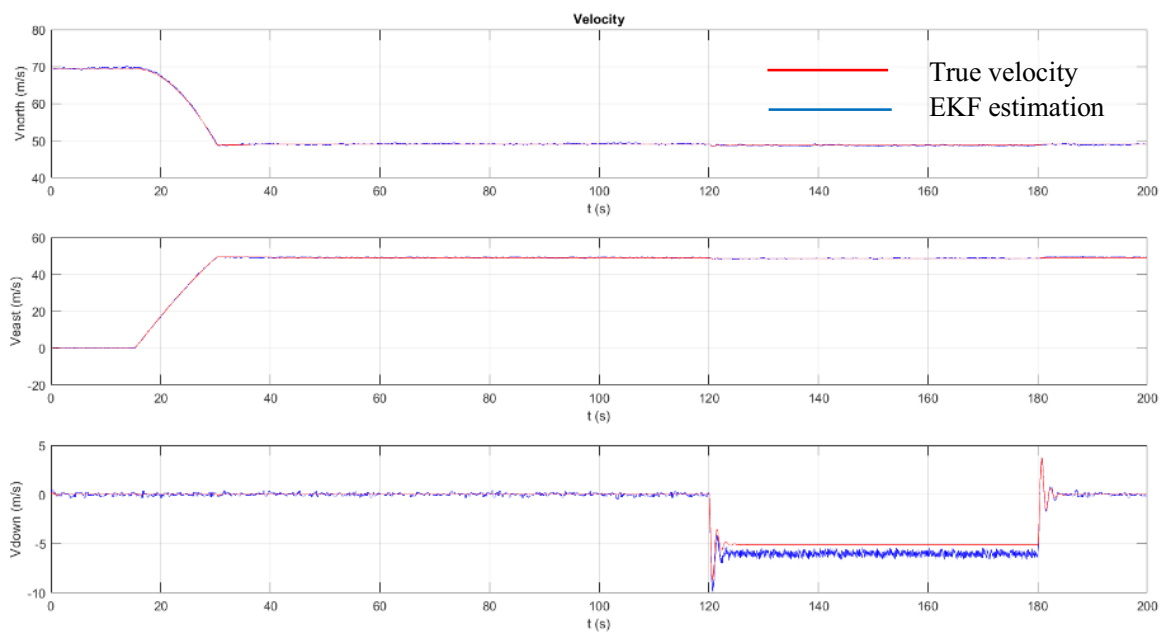

Figure 12. Aircraft velocity estimation.
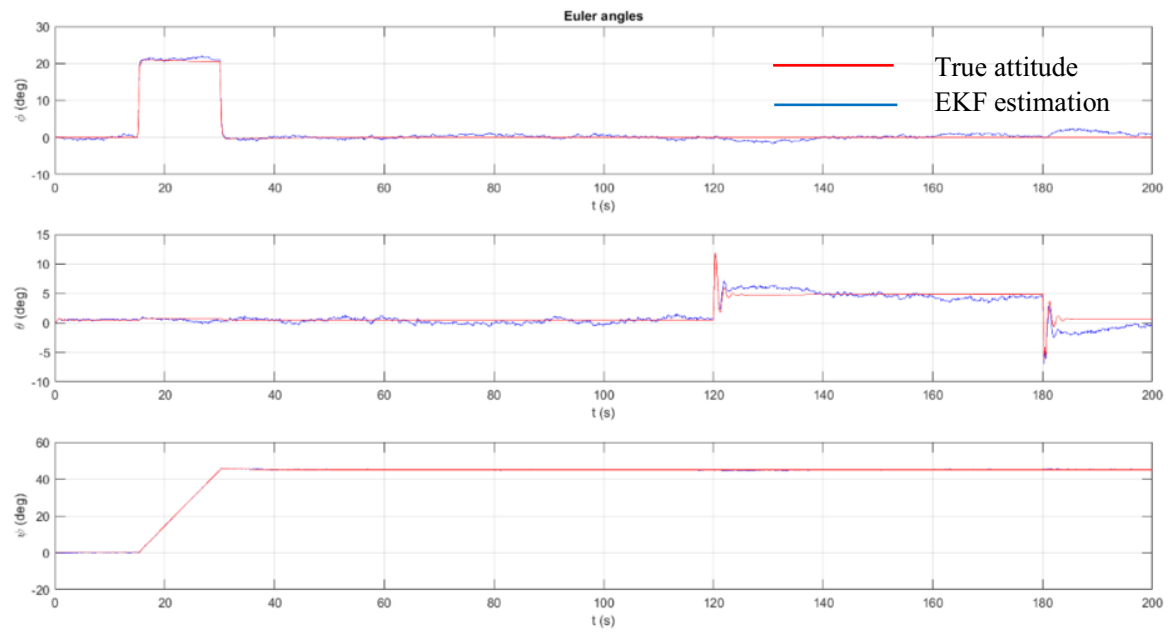

Figure 13. Aircraft attitude estimation.

$$
\begin{aligned}
& M_{a}^{b}=I_{b} \dot{\Omega}_{b}-M_{t}^{b}+\Omega_{b} \times I_{b} \times \Omega_{b} \\
& F_{a}^{b}=m a^{b}-F_{t}^{b}
\end{aligned}
$$

The values of $F_{a}^{b}$ and $M_{a}^{b}$ will be used for two tasks. The first task will be to feed a second Kalman based estimation algorithm in conjunction with the attitude estimated in the navigation EKF filter and the measured values of airspeed, angle of attack and sideslip from the ADS. The second task will be to train the aircraft digital twin model that will act as a virtual sensor and that it will also provide virtual values of $F_{a}^{b}$ and $M_{a}^{b}$ to the second EKF, aiding in the estimation of the airspeed, angle of attack, angle of sideslip and $w^{b}$ in the case of an ADS failure. 


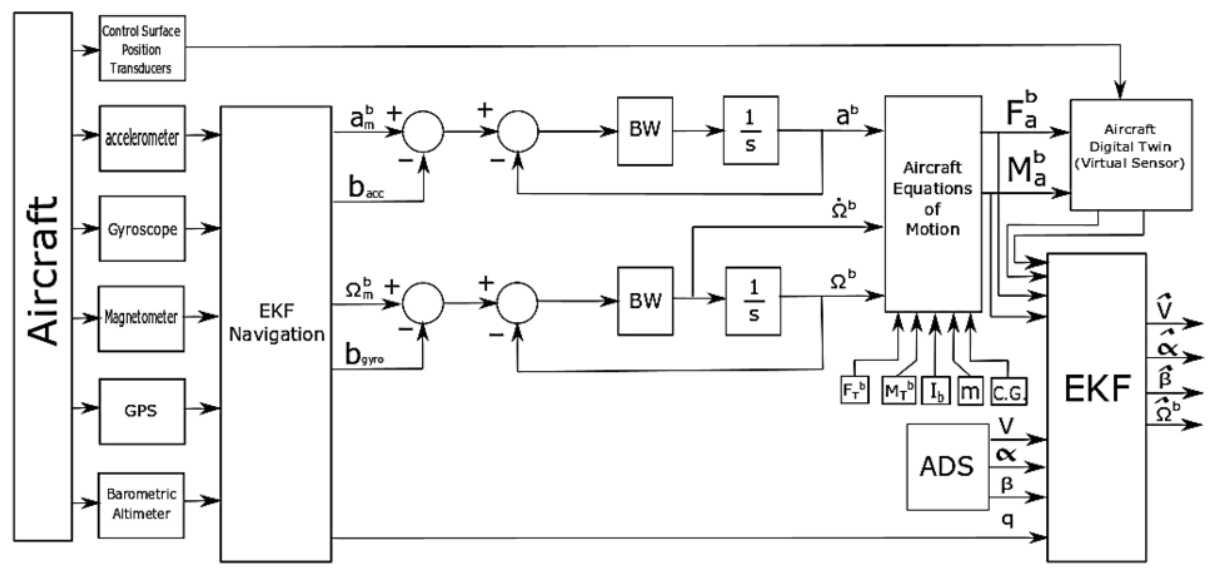

Figure 14. Proposed estimation filter.

\section{Conclusion}

This paper discusses different methods to estimate the aircraft airspeed and other state variables in case of a Pitot tube failure. The EKF navigation filter with sequential measurement update has shown to be a promising tool in the estimation of the airspeed, attitude and position with enough accuracy and low computational effort. The addition of adding sensors like the barometric altimeter to the estimation and the digital twin virtual sensor will increase the accuracy of the estimation filter especially in the estimation of the downward component of the velocity and the altitude. Higher GPS sample rates are desirable to decrease the time of the altitude estimation convergence. In addition a parametric study on the EKF estimation performance must be carried out varying the sensor parameters and noise.

\section{References}

[1] B. Stevens, F. Lewis and E. Johnson, Aircraft Control and Simulation, John Wiley \& Sons, Hoboken, 2016.

[2] M. R. Napolitano, Aircraft Dynamics: from Modeling to Simulation, John Wiley \& Sons, Hoboken, 2011.

[3] M.R. Napolitano, S. Cascianelli, S. Gururajan, M.B. Rhudy and M.L. Fravolini, Experimental Evaluation of Two Pitot Free Analytical Redundancy Techniques for the Estimation of the Airspeed of an UAV, SAE Int. J. Aerosp., 7(1):109-116, 2014.

[4] M.R. Napolitano, M.B. Rhudy, H. Chao, M.L. Fravolini and S. Gururajan, Performance Evaluation of Neural Network Based Approches for Airspeed Sensor Failure Accomodation on a Small UAV, 21st Mediterranean Conference on Control \& Automation (MED), 25-28 June 2013.

[5] S. Hussain, M. Mokhtar, J.M. Howe, Sensor Failure Detection Identification and Accommodation Using Fully Connected Cascade Neural Network, IEEE Transactions on Industrial Electronics, vol. 62, pp. 1683-1692, 2015.

[6] F. Balzano, M.L. Fravolini, M.R. Napolitano, S. d'Urso, M. Crispoltoni and G. del Core, Fault Detection With An Augmented Floating Limiter, International Journal of Aerospace Engineering, 2018, 1072056.

[7] S.M. Oh and E. Johnson, Development of UAV Navigation System Based on Unscented Kalman Filter, AIAA Guidance, Navigation, and Control Conference and Exhibit, August 2006, Keystone, Colorado. 